\title{
Three's company, or a crowd?: The effects of robot number and behavior on HRI in Japan and the USA
}

\author{
Marlena R. Fraune ${ }^{1}$, Satoru Kawakami ${ }^{2}$, Selma Šabanović ${ }^{1}$, P. Ravindra S. de Silva ${ }^{2}$, Michio Okada ${ }^{2}$ \\ ${ }^{1}$ Indiana University, ${ }^{2}$ Toyohashi University of Technology
}

\begin{abstract}
In everyday applications of robotics, people will likely interact with groups of robots. Most human-robot interaction (HRI) research to date, however, has studied humans interacting with individual robots. Initial research suggests that humans respond differently to individual robots and robots in groups, making responses to groups of robots critical to study. This paper presents a study performed in a public setting familiar to participants (university cafeterias) to examine how participants respond when robots, individually and in groups, enter their space. We examined participant survey and behavioral responses to different numbers of robots (Single or Group) with different behaviors (Social or Functional). Because robots will be used across cultures, we performed the study in Japan and the USA. Across cultures, we found that people interact more with robots in groups than single robots, yet report similar levels of liking for both; participants also rated social robots as more friendly and helpful than functional robots in general. They rated single social robots more positively than a group of social robots, but a group of functional robots more positively than single functional robots. Japanese participants reported liking the robots more than USA participants. This suggests that researchers and designers should be aware of how robot characteristics influence group effects.
\end{abstract}

Social human-robot interaction, group effects, robot behavior, cross-cultural study

\section{INTRODUCTION}

Many future robotics applications imply the use of multiple robots interacting with people and with each other (e.g, factory robots, several small robotic vacuums working in tandem [1]). In contrast, most human-robot interaction (HRI) research has to date focused on people interacting with individual robots, even when examining effects like group membership (e.g., [2]). Well-established social psychological theories predict group effects - that is, changes in attitude and behavior when people are exposed to or interacting with groups rather than individuals [3, 4]. As many important social psychological factors that determine the quality and quantity of social interactions have been successfully applied to HRI research (e,g., gaze [5, 6]), we can expect that group effects will also emerge in people's evaluations of and behaviors toward robots. Initial evidence suggests that responses to groups of robots may indeed differ from responses to individual robots [7]. However, this hypothesis has yet to be examined with people and robots interacting in a real-world setting.

This paper presents a study testing whether group effects paralleling those established in social psychology may be found in HRI - specifically, that one's attitudes and behaviors toward robots may change significantly when observing or interacting with an individual robot versus a group of robots. We specifically examined how Robot Number (Single, Group), Robot Behavior (Social, Functional), and Country of Interaction (Japan, USA) affected people's reactions to robots. We used minimally social robots to avoid strong effects of anthropomorphism and performed the study in public settings - university dining halls — in both countries.

\section{BACKGROUND}

\section{A. Robot Number and Group Effects in HRI}

HRI researchers have found that people often treat robots similarly to how they treat people in human-human interaction (HHI) [8] and that group effects from HHI may be applied to HRI. Sometimes, humans treat robots as ingroup or outgroup members depending on social cues (e.g., the robot's origin) [9], even in arbitrarily assigned groups [10]. These findings suggest that insights from social psychology on inter- and intra-group attitudes and behavior can be applied to HRI. However, in most studies examining group effects in robots, strong social cues are applied to the robots [3]. Most studies examine group effects for humanoid robots only (e.g., [9]), and provide further cues of the robots' anthropomorphism through various means: by assigning existing human social categories to robots (e.g., robots were said to be Turkish in [2]), or through the robot's humanlike appearance or behaviors (e.g., verbally greeting participants, supposedly having performed the task that participants would perform [9]).

Results from studies that cue anthropomorphism differ from results with mechanomorphic robots (e.g., iRobot Create). For example, competition increases when people interact in groups and against outgroups (e.g., [10]), but in one study of mechanomorphic robots, competition only increased when there were multiple human competitors, while there was no effect of number of robot competitors [4]. In another study, co-presence with a group of mechanomorphic robots (iRobot Create) did not affect attitudes toward robots, though it would have made attitudes more negative if group effects applied to the situation [3]. This suggests that cues of robot sociality and anthropomorphism-whether due to the robot's humanoid form, the social tasks the robot performs, the robot's social behaviors toward the experimenter and participants (e.g., 
[11]), or the experimenters' behavior toward the robotsincrease humans' social responses to robots. In this study, we sought to remove all cues but the robots' own characteristics including cues from the experimenters themselves. The study was performed in a public setting, with minimal intervention by experimenters, using voluntary interactions with passersby.

\section{B. Effects of Robot Appearance and Behavior on HRI}

Robot characteristics such as appearance and behavior are critical factors that determine if people perceive robots socially. Physical appearance can constrain a robot's social behavioral repertoire and thus can influence user expectations for social interactions. For example, anthropomorphic features can confer social advantages to robots by allowing them to engage in human social behaviors, such as emotional expressions and other human gestures [12], which can enhance social trust and positive social perceptions [6]. Studies suggest that a robot's behavior affected people's mental models of how to interact with it [13, 14]. Further, when a robot performed very social behavior (e.g., didactic tutorials, complimented participants), participants held the robot almost as accountable for its actions as they would a human and even ascribed mental, emotional, and social attributes to it [11].

In this study, we sought to use a robot with a form that was neither too anthropomorphic nor too mechanomorphic. A robot that was too anthropomorphic or even humanoid might automatically cue participants to respond to it as they would respond to humans (e.g., [9]). Conversely, a robot with too many mechanomorphic features that increase expectations for robots to perform mechanical tasks (e.g., fixing things), might receive fewer social benefits (e.g., [3]). Because studies suggest that a robot's appearance and behavior should match [6], a minimally social form should allow the robots to perform either social or nonsocial behavior without creating dissonance due to a mismatch between appearance and behavior. We used only nonverbal robot behavior (e.g., driving, turning, bowing/nodding) to manipulate the robots' sociality (see the Method Section III-A for details).

\section{Interactions between Robot Number and Robot Behavior}

Groups of robots may cue people to categorize the robots differently than when they are single. One study showed that when people viewed videos of robots, robot number affected attitudes toward robots differently depending on the robot's type (appearance and behavior) [7]. Human-like robots were more liked in groups than singly, but machine-like robots were less liked in groups than singly [7]. The differential results may be because people categorized the groups differently (e.g., "like me" or "like a robot"). Groups may induce different categorization because the group context can give cues regarding the social goals of groups members [10].

However, this may be true only when groups are social to begin with. In a study using groups of mechanomorphic robots performing nonsocial tasks unrelated to participants, no effect was observed on participants' subjective reports of how much

National Science Foundation, Grant Number DGE-0903495 they liked the robots, as would have been expected if the robots were seen as a social outgroup [3].

Differential attitudes based on viewing single or groups of robots has yet to be demonstrated during actual interaction. In this study, we expect that the robots' behavior may interact with number of robots, and even number of people, to affect how people respond to the robots.

\section{Cultural Context in HRI}

Previously, researchers suspected that participants from Western cultures (e.g., USA) would respond more negatively toward robots than those from Eastern cultures (e.g., Japan), due to the more-negative portrayal of robots in the media in Western than Eastern cultures [15] and negative stereotypes about robots in the West (e.g., "Robots will take my job") [16]. However, some cross-cultural comparisons suggest fewer differences than expected based on implicit and explicit measures of attitudes toward robots [17, 18].

However, Eastern and Western people show concern and interest about different aspects of robots. Japanese participants more often mentioned negative sentiments about emotional or social issues, concern about robots performing anti-social behavior, and wanting robots to fit into society [19]. Conversely, participants from the UK were more likely to state acceptance of robots as long as they were not too human-like [19]. In an open interview, Eastern (Korean) participants showed preference for friendly, social, human-like robots, while USA participants preferred stylish or modern robots to use as functional tools [20,21]. Empirical studies also suggest that Easterners tend to prefer small, interactive robots more than do Westerners, indicating cultural influence on robot preferences [22]. In the present study, Easterners may prefer more social robots than Westerners.

\section{E. Cultures, Groups, and Behavior of Robots}

Across cultures, group and social cues have differing importance in how they influence interactions. For example, people from more Collectivistic or Low-Context cultures (e.g., Japan) place higher importance on social cues [23], even social cues from robots, than do people from more Individualistic or High-Context (e.g., USA) [24]. One study showed that robot appearance (small anthropomorphic, zoomorphic, or mechanomorphic robots) did not interact with cultures, but that Western (German) participants were more interactive with robots on social than nonsocial tasks, whereas Eastern (Chinese, Korean) participants were highly interactive with robots on all tasks [22].Thus, culture appears to influence how people conceptualize and respond to robots, singly and in groups. In the end, it will be most useful to have robots that can traverse cultural boundaries and be accepted in multiple cultures. In this study, we examine how specific robots (Sociable Trash Boxes) are viewed in both Japan and the USA. To examine if a robot with fewer or more minimalistic social cues might be acceptable across cultures with different levels of Individualism/Collectivism and High/Low Context, we chose a robot that has some functional purpose and (in the 
social conditions) shows only minimalistic, nonverbal social cues (e.g., nodding/bowing, contingent responses).

\section{F. Lab and Public Settings for HRI Research}

Most HRI experiments occur in lab settings (e.g., [2, 4, 7]), though recently HRI studies in naturalistic, everyday settings are becoming more common (e.g. nursing homes [25], malls [26]). Studies suggest that when participants are outside of their regular comfort zone, such as when they are in a laboratory, they are more accepting of others and seek more to fit in, contrasted with a greater likelihood to reject others when they remain in a familiar place [27]. This may introduce a confound in studies - and in fact, some HRI studies illustrate that participants are more positive and accepting toward robots when they are in the lab than before they enter the lab [3]. To understand how people respond to robots in a familiar place that is part of participants' daily lives, we placed robots in university cafeterias, where students eat multiple times per week and are familiar with the environment. The robots were placed there as trash bins, which gave them a believable and contextually appropriate task to perform.

\section{G. Overview}

We examine the effects and interactions of three factors on HRI in a public setting: (1) Number of Robots (Single, Group) - one robot or three robots in the cafeteria at once, (2) Behavior of Robots (Social, Functional) - social robots responded contingently to humans and robots, but functional did not, and (3) Country (Japan, USA) - place of study.

For this study, we used minimally social robots (see Figures 1 and 2), which humans could easily construe as social or functional depending on the type of nonverbal interactional cues provided by the robot. The study tested the following hypotheses regarding interactions between people and robots:

H1. When the robots are in groups (rather than single), participant reactions will become more extreme (e.g., if they interact with one type of robot, they will interact even more with a group of that type of robot).

H2. Participants will prefer social robots to functional robots: People will interact for longer, throw more trash in robots, and rate robots more positively.

H3. Participants in Japan will interact more with robots and show more liking for robots than participants in the US.

H4. Participants in Japan will prefer social robots, and participants in the USA will prefer functional robots.

\section{METHOD}

The field study took place in cafeterias at Toyohashi University of Technology and Indiana University. There, the Sociable Trash Box robots (STBs; Figures 1 and 2) patrolled near trash and recycling bins, as alternative receptacles for trash/recycling. The study used a 2 Number of Robots (Single, Group) x 2 Behavior of Robots (Functional, Social) x 2 Country (Japan, USA) design.

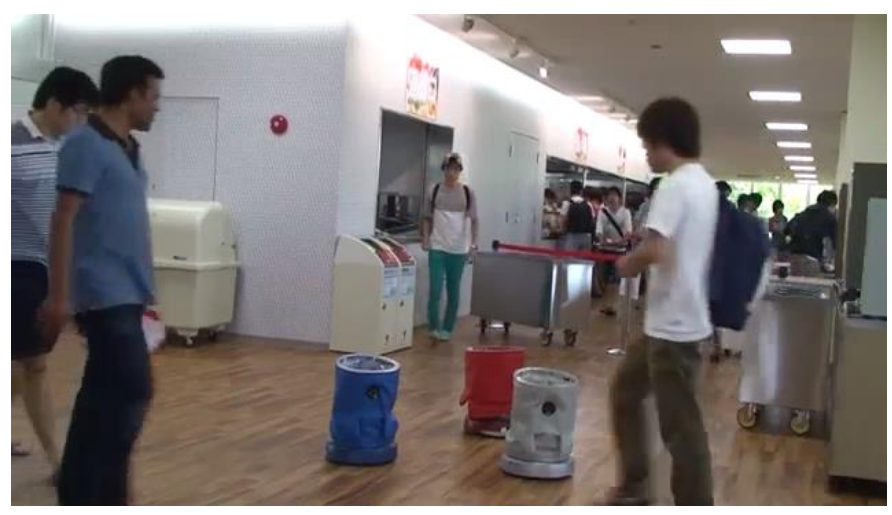

Figure 1. Sociable Trash Box robots (STBs) in Japan

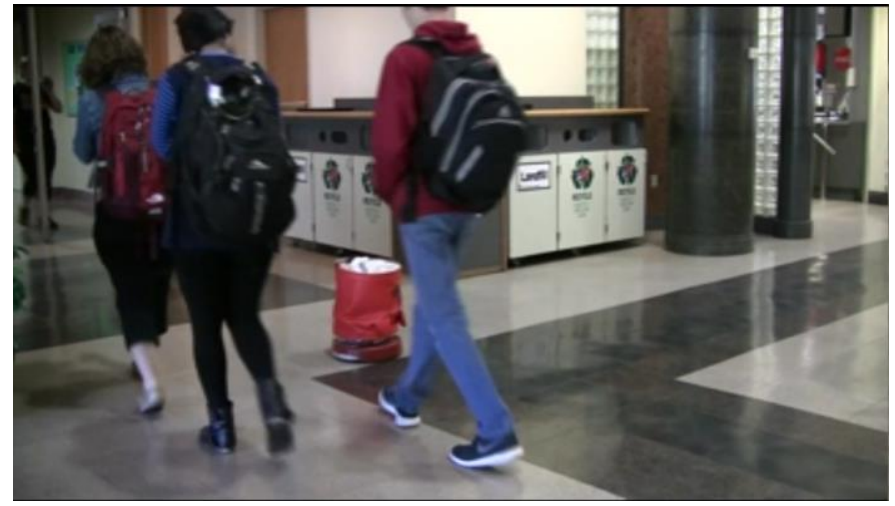

Figure 2. Sociable Trash Box robots (STBs) in the USA

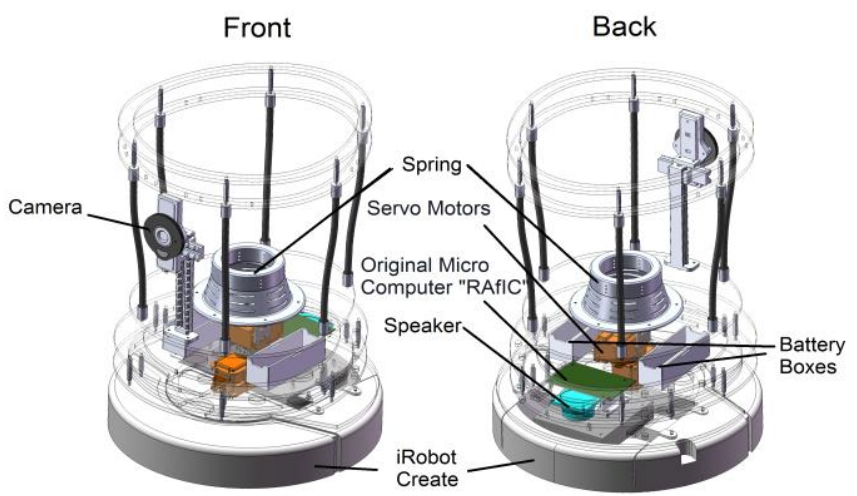

Figure 3. STB Design

\section{A. Robot Platform}

Attempting to use a "minimally social" robot, as discussed in Section II-B, we used the Sociable Trash Box (STB) robots (Figures 1 and 2) [28]. These robots have anthropomorphic traits (e.g., eye, arms), but are still far from humanlike. The STBs have no legs, but their motion is a gentle left-and-right that mimics swaying back and forth from small steps forward. We expected that these minimal social cues would be enough to not overly bias participants to respond socially or nonsocially toward the robots, allowing us to examine the effects of manipulating only the robots' behavior.

The STB platform included robots on iRobot Create bases (Figure 3). The RAfiC computer controlled movement (e.g., 
driving) and used three Servo Motors for other behavior (e.g., bowing, looking). Human drivers communicated with Android phones through an internet server to control the STBs.

\section{B. Procedure}

The study was IRB-approved. Each condition was run three times for approximately one hour during regular mealtimes (i.e., breakfast, lunch, dinner) for a total of 24 sessions. Conditions were run in a counterbalanced order to account for order effects. Robots were controlled via the Wizard of Oz (WOZ) technique, with one person controlling each robot. The robots' drivers sat in the cafeteria in order to view the designated area. Drivers in Functional conditions were told to make the robots approach individuals for five seconds then depart regardless of the participant's behavior. Group Functional robots moved independently of each other. Drivers in Social conditions were told that the robots should approach people and bow/nod at them as a "greeting" and also as a nonverbal "thank you" for throwing trash in them. Social robots responded to participants contingently. In the Group Social condition, drivers were also told that the robots should occasionally interact with each other (e.g., turn toward each other, bow/nod at each other), particularly when there were few participants passing. Robots typically remained about 50 centimeters from humans and approximately one meter from furniture to prevent blocking participants' paths.

To prevent people from guessing that experimenters were controlling the robots, experimenters brought food and used a smartphone or tablet to control the robots. This matched the behavior of students in the area, who sit in one place for a long time on a smartphone or tablet. When interacting with the robots, participants seldom looked at the experimenters, suggesting that they usually did not know the robots were being controlled remotely or that a study was taking place.

The study area was (visibly) videotaped, with focus on the interactions people had with the robot, and the video was later coded for participant behavior (time they looked at, interacted with, and threw trash in the robots). With inter-rater reliability of $74-82 \%$, coders displayed moderate to high reliability (see Section IV-B for details). An assistant approached individuals after they left the monitored area to ask for consent and administer the survey to those who agreed to participate.

\section{Materials}

The post-interaction survey questioned participants about the robots that they saw the same day. It included 7-point Semantic Differential questions about the robots' traits (Negative/Positive, Scary/Friendly, Mean/Kind, Useless/ Useful, Stupid/Smart, Nonsocial/Social, Machine-like/Humanlike), how participants would feel if someone kicked the robot (Sad/Happy), if they felt the robot could help them, if they could help the robot, if they would use the robot to recycle (or sort trash), if they recycle (or sort trash) in general, if they would be proud if the robots were well-known, and if they wanted the robots at their school.

\section{RESULTS}

Data were analyzed in SPSS, and $p$-values of .050 or less were considered significant. Bonferoni corrections were used for multiple comparisons. For details, see Table 1 about participant behaviors and Table 2 about survey responses.

\section{A. Survey Results}

The survey was administered directly after participants exited the dining areas where the robots were located (141 in Japan, 120 in USA). A series of 2 (Number: Single, Group) x 2 (Behavior: Social, Functional) x 2 (Culture/Country: Japan, USA) ANOVAS were run on survey responses.

A main effect of Number occurred such that participants thought they could help Single robots more than Group robots $\left(F(1,253)=9.78, p=.002, \eta^{2}=.039\right)$. A main effect of Behavior occurred, such that participants rated Social robots as more Friendly (as opposed to scary) than Functional robots $\left(F(1,241)=9.66, p=.002, \eta^{2}=.040\right)$, and they thought Social robots could help them more than Functional robots $\left(F(1,253)=4.25, p=.004, \eta^{2}=.017\right)$. A main effect of Country occurred such that Japanese participants reported that they could help the robots more than USA participants did $\left(F(1,253)=20.27, p<.001, \eta^{2}=.080\right)$. Japanese participants reported that they would be more sad if someone kicked the robot than USA participants $\left(F(1,253)=8.78, p=.003, \eta^{2}=\right.$ .035). Japanese participants reported both that they recycle in general less $\left(F(1,253)=7.03, p=.009, \eta^{2}=.028\right)$ and would use the robot for recycling less $\left(F(1,253)=27.31, p<.001, \eta^{2}\right.$ $=.108$ ) than did USA participants.

\begin{tabular}{|c|c|c|c|c|c|c|c|c|}
\hline Country & $\begin{array}{c}\text { Robot } \\
\text { Number }\end{array}$ & $\begin{array}{c}\text { Robot } \\
\text { Behavior }\end{array}$ & $\begin{array}{c}\text { Passed By } \\
(N)\end{array}$ & $\begin{array}{c}\text { Looked }>2 \\
\sec (\%)\end{array}$ & $\begin{array}{c}\text { Interacted } \\
(\%)\end{array}$ & $\begin{array}{c}\text { Threw Trash } \\
(\%)\end{array}$ & $\begin{array}{c}\text { Trash in STB } \\
(\%)\end{array}$ & $\begin{array}{c}\text { Took Survey } \\
(N)\end{array}$ \\
\hline \multirow[t]{4}{*}{ Japan } & \multirow[t]{2}{*}{ Single } & Functional & 719 & 53.6 & 5.0 & 17.5 & 4.3 & 26 \\
\hline & & Social & 523 & 28.9 & 1.0 & 14.9 & 3.4 & 38 \\
\hline & \multirow[t]{2}{*}{ Group } & Functional & 620 & 37.7 & 3.9 & 12.6 & 5.8 & 32 \\
\hline & & Social & 280 & 24.3 & 6.1 & 10.4 & 2.1 & 45 \\
\hline \multirow[t]{4}{*}{ USA } & \multirow[t]{2}{*}{ Single } & Functional & 631 & 19.2 & 0.5 & 5.7 & 0.5 & 30 \\
\hline & & Social & 680 & 26.9 & 1.0 & 5.9 & 0.6 & 30 \\
\hline & \multirow[t]{2}{*}{ Group } & Functional & 449 & 40.5 & 1.3 & 5.4 & 0.7 & 30 \\
\hline & & Social & 231 & 37.7 & 1.7 & 8.2 & 1.3 & 30 \\
\hline
\end{tabular}




\begin{tabular}{|c|c|c|c|c|c|c|c|c|c|c|c|c|c|c|c|c|}
\hline $\begin{array}{l}\text { Coun- } \\
\text { try }\end{array}$ & $\begin{array}{l}\text { Robot } \\
\text { Num }\end{array}$ & $\begin{array}{l}\text { Robot } \\
\text { Beh }\end{array}$ & $\begin{array}{l}\text { Negative } \\
\text { /Positive }\end{array}$ & $\begin{array}{l}\text { Scary/ } \\
\text { Friendly }\end{array}$ & $\begin{array}{l}\text { Mean/ } \\
\text { Kind }\end{array}$ & $\begin{array}{l}\text { Useless/ } \\
\text { Useful }\end{array}$ & $\begin{array}{l}\text { Stupid/ } \\
\text { Smart }\end{array}$ & $\begin{array}{l}\text { Non- } \\
\text { /Social }\end{array}$ & $\begin{array}{l}\text { Like a } \\
\text { Machine } \\
\text { /Human }\end{array}$ & $\begin{array}{l}\text { Sad/ } \\
\text { Happy if } \\
\text { kicked }\end{array}$ & $\begin{array}{l}\text { Robot } \\
\text { could } \\
\text { help me }\end{array}$ & $\begin{array}{l}\text { I could } \\
\text { help } \\
\text { robot }\end{array}$ & $\begin{array}{l}\text { I usually } \\
\text { Recycle }\end{array}$ & $\begin{array}{l}\text { Would } \\
\text { use to } \\
\text { Recycle }\end{array}$ & $\begin{array}{l}\text { Would } \\
\text { be proud }\end{array}$ & $\begin{array}{l}\text { Want } \\
\text { school } \\
\text { to have }\end{array}$ \\
\hline \multirow[t]{4}{*}{ Japan } & Single & Funct & $5.0(0.9)$ & 4.1(1.6) & $4.8(1.3)$ & $4.2(1.3)$ & $4.2(1.1)$ & $4.3(1.5)$ & $2.7(1.4)$ & $2.0(1.2)$ & $3.8(1.4)$ & $4.2(1.4)$ & $6.1(1.0)$ & $4.1(1.6)$ & $5.5(1.2)$ & $5.4(1.3)$ \\
\hline & & Social & $5.3(1.2)$ & $4.7(1.2)$ & $4.7(1.1)$ & $5.2(1.3)$ & $4.9(1.3)$ & $4.8(1.0)$ & $4.0(1.4)$ & $1.9(1.0)$ & $4.4(1.3)$ & $4.4(1.1)$ & $5.3(1.4)$ & $4.8(1.5)$ & $5.7(1.0)$ & $5.7(1.1)$ \\
\hline & Group & Funct & $5.1(1.1)$ & $4.4(1.6)$ & $4.8(1.4)$ & $4.8(1.0)$ & $4.7(1.6)$ & $4.9(1.3)$ & $3.3(1.3)$ & $2.0(1.0)$ & $4.0(1.6)$ & $4.4(1.0)$ & $5.9(1.2)$ & $4.6(1.7)$ & $5.8(1.1)$ & $5.6(1.3)$ \\
\hline & & Social & $5.3(1.1)$ & $5.0(1.4)$ & $4.6(1.2)$ & 4.3(1.1) & $4.2(1.2)$ & $4.4(1.3)$ & $3.1(1.3)$ & $1.7(1.4)$ & $4.0(1.6)$ & $3.9(1.4)$ & $5.5(1.4)$ & $3.9(1.7)$ & $5.6(1.1)$ & $5.0(1.8)$ \\
\hline \multirow[t]{4}{*}{ USA } & Single & Funct & $5.1(1.2)$ & $4.8(2.0)$ & $4.4(2.1)$ & 4.4(1.7) & 4.4(1.8) & $4.4(1.5)$ & $3.2(1.9)$ & $2.5(1.3)$ & $4.3(1.4)$ & $4.0(1.3)$ & $5.3(1.6)$ & 5.1(1.4) & $4.9(1.1)$ & $4.7(1.6)$ \\
\hline & & Social & $5.5(1.4)$ & $5.4(1.9)$ & $4.7(2.1)$ & 4.7(1.6) & 4.6(1.6) & $4.5(1.6)$ & $3.3(1.8)$ & $1.9(1.2)$ & $4.5(1.5)$ & $3.8(1.7)$ & 5.3(1.4) & $5.4(1.8)$ & $5.4(1.4)$ & $5.2(1.6)$ \\
\hline & Group & Funct & $5.6(1.2)$ & $4.3(2.3)$ & $4.5(2.3)$ & $5.0(1.7)$ & $4.9(1.8)$ & $4.4(2.0)$ & $3.7(2.0)$ & $2.0(1.2)$ & $3.9(1.6)$ & $2.7(1.7)$ & $5.0(1.7)$ & $5.9(1.3)$ & $4.6(1.8)$ & $5.4(1.5)$ \\
\hline & & Social & $5.3(1.2)$ & $5.2(1.6)$ & $5.0(1.8)$ & $4.7(1.5)$ & $4.4(1.7)$ & $4.4(1.8)$ & 3.1(1.8) & $2.9(1.3)$ & $4.7(1.4)$ & $3.2(1.6)$ & $5.3(1.6)$ & $5.2(1.7)$ & $5.1(1.2)$ & $5.0(1.9)$ \\
\hline
\end{tabular}

Table 2. Means and Standard Deviations of answers to survey questions by condition

Number and Behavior interacted for how Useful $(F(1$, $\left.239)=7.40, p=.007, \eta^{2}=.031\right), \operatorname{Smart}(F(1,238)=6.64, p=$ $\left..011, \eta^{2}=.028\right)$, and Human-like $(F(1,240)=5.97, p=.015$, $\left.\eta^{2}=.025\right)$ the robot was rated, for how likely participants were to use the robot to recycle $\left(F(1,252)=8.40, p=.004, \eta^{2}\right.$ $=.033)$, how sad participants would feel if someone kicked the robot $\left(F(1,253)=4.22, p=.041, \eta^{2}=.017\right)$, and how much participants wanted the robot in their schools $(F(1,253)=$ $\left.12.96, p=.020, \eta^{2}=.022\right)$. On these questions, participants in both countries rated Single Social robots more highly than Single Functional, and Group Functional more highly than Group Social (see Figure 4).

Number and Country interacted such that Japanese participants reported that they could help Single and Group robots about equally, but USA participants reported that they could help Single robots more than Group robots $(F(1,253)=$ 4.74, $p=.003, \eta^{2}=.019$ ).

Number, Behavior, and Country interacted $(F(1,253)=$ 6.89, $\left.p=.009, \eta^{2}=.027\right)$, such that Japanese participants reported similar levels of sadness for all robots being kicked, but USA participants reported that they would be more sad if the Single Social or Group Functional robots were kicked than if the Group Social or Single Functional robots were kicked.

No significant differences were found for how Positive/ Negative, Mean/Kind, or Social/Nonsocial participants found the robots, and their Pride if the robots became well-known.

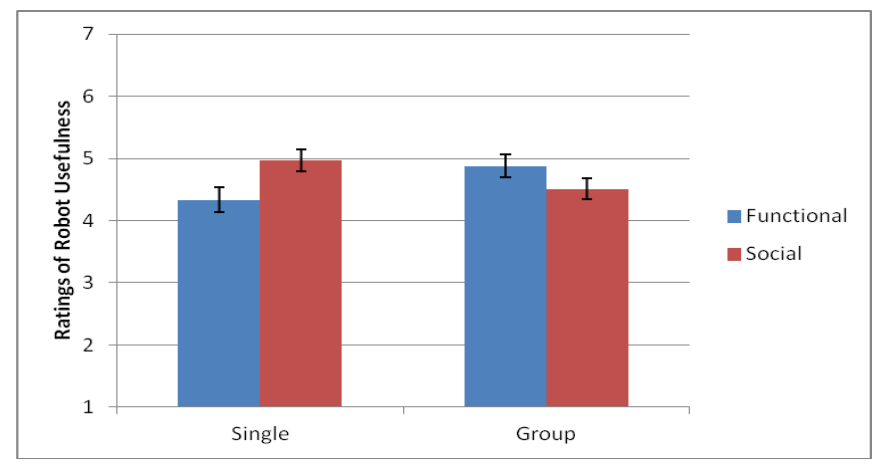

Figure 4. Typical interaction of robot Number and Behavior. Error bars indicate standard error.

\section{B. Video Results}

Coders rated videos of the space surrounding the robots and ensuing interactions. These videos totaled approximately five hours in Japan and five hours in the USA (total participants who walked past were 2067 in Japan, 2065 in the USA; see Table 1 for details). Participant behavior was coded for Look Duration (time spent looking at the robots), Direct Interaction (e.g., waving a hand toward the robots, touching them), and Trash in Robot (throwing trash in the STBs). To control for how many people had trash to throw away, throwing trash in the trashcan (not the STB) was also recorded. Participants were also coded as Single or Group entities based on interactions with other humans while on video (e.g., talking, direct eye contact, close coordination of motion). To calculate inter-rater reliability, video coding overlapped for $10 \%$ of videos (1.2 hours distributed across conditions) and were found to be $79 \%$ consistent. (Specific behavior - Look Time: 74\%, Direct Interaction: 77\%, Trash in Robot: $82 \%$, Trash in Can: $78 \%$ ). Coders displayed moderate to high reliability (74-82\%).

Looking Duration (seconds) differed depending on conditions (Figure 5), as determined by a 3-way ANOVA. Duration was skewed to the right, and to account for this, we took the $\log$ of the durations (plus 1 to prevent the error of taking the log of 0). Because there were large differences in duration for participants in Japan and in the USA, we divided the groups, and then excluded outliers from each group (4 for Japan and 17 for USA). Then we combined results again and ran a 3-way ANOVA on the results. (ANOVA results before and after outliers were excluded showed similar significant differences.) These results showed no main effect of robot Number $(F(1,4101)=3.30, p=.069)$, but an effect of Behavior $\left(F(1,4101)=24.36, p<.001, \eta^{2}=.006\right)$ indicated that people looked longer at Functional than Social robots. There was a main effect of Country $(F(1,4101)=73.72, p<$ $\left..001, \eta^{2}=.015\right)$, with participants from Japan looking at the robots longer in general than participants from the USA. Twoway interactions occurred (Country and Number, Country and Behavior), but to save space, we will report the more explanatory 3-way interaction between Country, Number, and Behavior $\left(F(1,4101)=62.92, p<.001, \eta^{2}=.013\right)$. Japanese participants looked longer at Group than at Single Social robots, but shorter at Group than at Single Functional 
robots. Conversely, USA participants looked longer at Group than at Single Functional robots and slightly shorter at Group than Single Social robots.

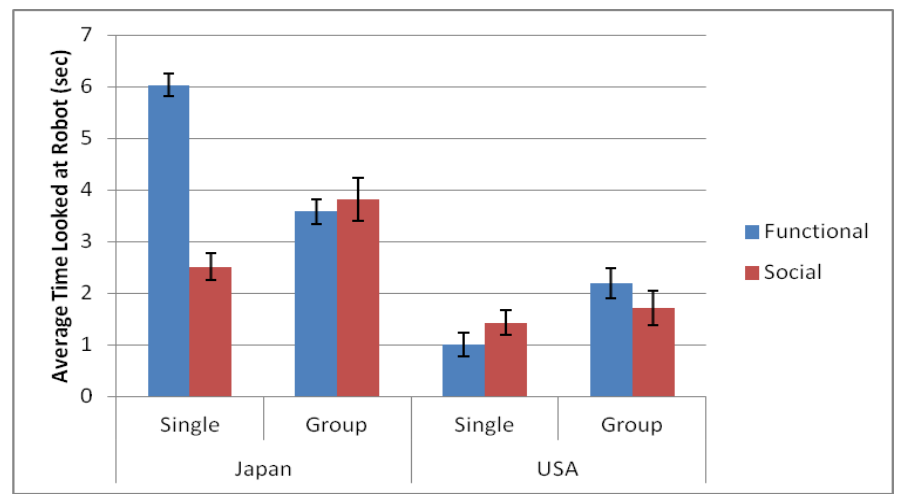

Figure 5. Difference in duration looking at robots. Error bars indicate standard error.

Direct interaction with robots showed differences according to conditions (Figure 6). Because very few people performed these behaviors, we analyzed the data as nominal variables and performed chi squared tests on whether or not people performed behaviors indicating direct interaction with robot (e.g., waving a hand or trash toward them, touching them, kicking or pretending to kick them). For participants overall, there was a main effect of Number such that more participants directly interacted with Group than Single robots $\left(X^{2}(1,3905)=1.94, p=.001\right)$. There was no main effect of robot Behavior $\left(X^{2}(1,3905)=0.90, p=.405\right)$. There was a main effect of Country, with more participants from Japan than the USA directly interacting with robots $\left(X^{2}(1,3905)=\right.$ $31.64, p=.001)$. Number and Behavior interacted such that participants interacted more with Group Social than Single Social robots $\left(X^{2}(1,3905)=41.29, p<.001\right)$, but there was no difference between Group and Single Functional robots $\left(X^{2}(1\right.$, $3905)<0.00, p=.980$ ). An interaction between Country and Number occurred such that for Japan, there was no significant difference between Single and Group robots $\left(X^{2}(1,3905)=\right.$ $3.60, p=.058)$, but for the USA, people interacted more with Group than Single robots $\left(X^{2}(1,3905)=4.51, p=.034\right)$. No interaction of Country and Behavior was found (Japan: $X^{2}(1$, $3905)=2.68, p=.101$; USA: $\left.X^{2}(1,3905)=2.31, p=.128\right)$. The three-way interaction could not be calculated due to an expected count of $<5$ for some cells in the chi squared test.

Of participants who threw trash away $(N=430)$, conditions affected who threw trash in the robots, as indicated by chi squared tests (Figure 7). (Only participants who threw trash away were included in this test because other participants often had no trash.) Of participants who threw trash away, $24 \%$ threw some in the robots. There was a main effect of Number such that participants were more likely to throw trash in Group than Single robots $\left(X^{2}(1,430)=7.67, p=.006\right)$. There was a main effect of Behavior, such that people were more likely to throw trash in Functional than Social robots $\left(X^{2}(1,430)=4.48, p=.034\right)$. A main effect of Country showed that participants in Japan were more likely to throw trash in the robots than participants in the USA $\left(X^{2}(1,430)=\right.$
$15.78, p<.001)$. An interaction occurred for Number and Behavior such that participants threw more trash in Group than Single Functional robots $\left(X^{2}(1,430)=9.31, p=.002\right)$, but there was no difference in Number for Social robots $\left(X^{2}(1\right.$, 430) $<0.00, p=.987)$. An interaction occurred between Country and Number such that Japanese participants threw more trash in Group than Single robots $\left(X^{2}(1,430)=7.87, p=\right.$ $.005)$, but no differences for Group and Single was found in the USA $\left(X^{2}(1,430)=0.64, p=.426\right)$. No interaction of Country and Behavior occurred (Japan: $X^{2}(1,430)=3.68, p$ $=.055$; USA: $\left.X^{2}(1,430)=0.11, p=.744\right)$. The three-way interaction could not be calculated due to an expected count of $<5$ for some cells in the chi squared test.

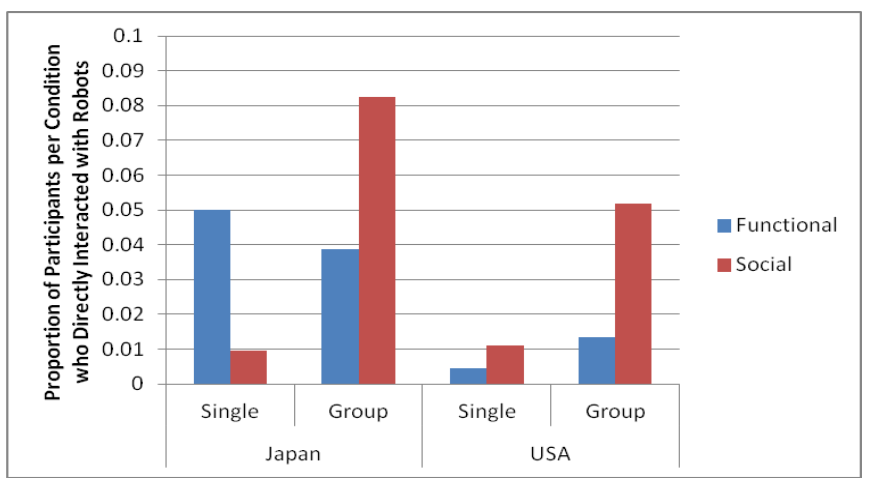

Figure 6. Ratio of participants who interacted with robots.

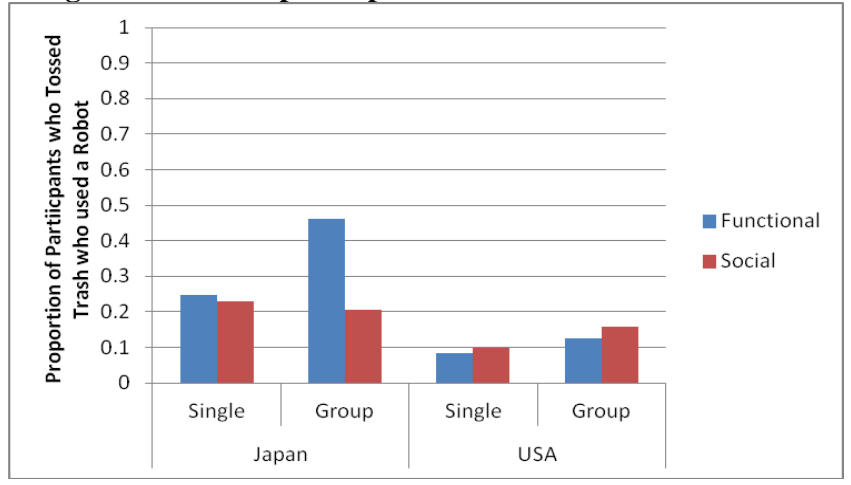

Figure 7. Ratio of participants who threw trash in STB robots.

Participants were also coded as Single or Group members based on their interactions with other people in the study area. Because this variable was challenging to code and potentially a product of interacting with people while interacting with the robots, we did not examine it in relation to independent variables. This preliminary data suggested that Groups of Humans looked for a longer time at robots $\left(X^{2}(2,3974)=\right.$ $442.76, p<.001)$, performed more direct interaction with robots $\left(X^{2}(2,3905)=54.22, p<.001\right)$, and were more likely to throw trash in the robots $\left(X^{2}(2,430)=25.45, p<.001\right)$.

\section{DISCUSSION}

Overall, the survey data suggests that across cultures, people preferred Single Social robots and Group Functional robots to Single Functional or Group Social robots. This occurred across several survey measures and showed 
interesting interactions with behavioral data. In this discussion, we will report survey and behavioral data together. First we detail main effects of Number, Behavior, and Country, in relation to survey and behavioral responses of participants, and then we examine the interaction effects.

\section{A. Main Effects}

Robot Number had little effect on survey responses, or time looking at the robots, but it did affect how people interacted with the robots. Groups of robots induced more direct interaction and more trash being thrown in them, partially supporting H1 (i.e., people may interact more with groups). This may be explained by greater numbers of robots giving more space for participants to directly interact with them, whereas looking duration depends less on being near the robots. Because the survey data indicated that the number of robots alone did not affect participant liking of the robots, robot number could be increased for practical applications without incurring more explicit negative attitudes toward them. However, it is important to understand how the number of robots may interact with the robots' characteristics (e.g., behavior, appearance) to affect attitudes toward the robot.

Robot Behavior affected survey and behavioral responses to the robots. Participants found Social robots overall to be more friendly and helpful than Functional robots (lending partial support to $\mathrm{H} 2$ that people prefer Social to Functional robots). Functional robots, however, elicited longer looking duration and more trash was thrown in them. This may have been because Social robots often spent time socializing among themselves and with participants, which sometimes drew them away from other passing participants. This suggests that robots need not be "liked" more in order for them to successfully perform functional tasks; in fact it might be better if they are less likeable and socially interactive.

Country affected survey and behavioral responses to the robots. In some responses (i.e., pride, sad if someone kicked the robot), Japanese participants showed more positive evaluations of robots. Related qualitative video data showed that no participants in Japan kicked the robot, whereas several participants in the USA either kicked or pretended to kick the robots (sometimes resulting in broken parts). This may be interpreted to suggest that the data somewhat support $\mathrm{H} 3$ (Japanese participants are more positive toward robots than USA participants). Survey and behavioral findings on responses to kicking the robots may also relate to cultural norms on respect for technology or property, or in performing potentially violent behavior. Behaviorally, Japanese participants looked at robots longer, performed more direct interaction, and threw more trash in the robots than USA participants. However, this variability may relate to variability in locations. In Japan, participants had to stand in line to put away their lunch trays and dishes, making them wait longer near the robots, whereas in the USA, very few participants had to wait to put their lunch materials away, meaning that they could walk past the robots more quickly and interact less.
Participant Number (single or group) was preliminarily examined in relation to participant behavior toward the robots. People in groups looked at the robots longer, performed more direct interaction with the robots, and threw more trash in the robots than did single humans. However, the video data did not allow us to determine why this was. One hypothesis is that humans in groups drew support from group members [29], making them more willing to interact with unfamiliar robots. Conversely, it may be that the robots, by drawing people's attention, induced them to interact more. Qualitatively, video coders reported that if one person stopped to interact with the robot, other people also tended to stop to interact, looking at the robots and at other human actors during the interactions. This social mediating effect also occurred in prior work with the socially assistive Paro robot in nursing homes [25]. While this data is inconclusive, it underlines the importance of studying bidirectional effects of groups of humans and robots interacting.

\section{B. Interaction Effects}

Interactions between Number, Behavior, and Country were significant. Survey data suggest that across cultures, people preferred Single Social robots and Group Functional robots to Single Functional or Group Social robots. Our results therefore do not support H4 (Japanese participants would prefer social robots and USA participants would prefer functional robots). Specifically, this occurred in relation to how useful, smart, and human-like the robots were, how sad they would be if they saw someone kick the robots, how much they wanted them in their school, and how likely they were to use them for recycling. One possible explanation for this is that groups of robots confer presumed sociality to each other [7]. When the robots behaved socially while in a group, it may have had an additive effect on the sociality of the group, making them appear as part of their own group. Participants may interpret a cohesive group of social robots as an outgroup [10], inducing more negative attitudes toward them. In contrast, when the robots behaved functionally while in a group, their behavior might decrease the perceived sociality of the robots. This might make them engaging but not social enough to be perceived as a potentially-threatening outgroup, therefore inducing more positive attitudes. Similarly, single robots would not have the group-conferred sociality, so the single social robot might be social enough to be engaging, but not social enough to be perceived as an outgroup member, thus inducing more positive responses. In contrast, the single functional robot would not gain sociality from group status or from its own behavior, which could make it seem less engaging and may induce more negative attitudes toward it.

These findings show disparity in relation to other group HRI findings, in which participants who watched robot videos preferred more social (humanoid) robots in a groups than individually and preferred less social (mechanomorphic) robots individually than in a group [7]. The divergence may be caused by robots entering places participants were familiar with (university cafeterias) in this study rather than being viewed by participants in videos. The former may be more 
threatening than the latter. It may also relate to how humanlike the behavior is, with more human-like group behavior such as waving ([7]) being preferred over less-human-like nodding/bowing group behavior used in this study. These findings illustrate the need to further examine responses to robot groups, characteristics, and interaction styles both in and outside of the laboratory setting.

Critically, with regards to behavioral responses to robots, a 3-way interaction was found showing that Japanese participants tended to look for longer durations at Single Functional than Single Social robots and for longer at Group Social than Group Functional robots. Conversely, USA participants looked for longer periods at Single Social than at Single Functional robots and for longer at Group Functional than Group Social robots. Similar patterns were found for interacting with the robots. When compared to participant selfreports of how much they liked the robots, this suggests that Japanese participants looked longer at and interacted more with robots they liked less and USA participants looked longer at and interacted more with robots they liked more. Because specific participants in the video cannot be matched with participants who completed surveys, these data cannot directly support this trend. We also do not know in which direction this interaction occurred. It may be that because participants in each country liked or disliked the robots, they looked at and interacted with the robots for longer or shorter durations. It may also be that looking at the robots for longer or shorter durations, or interacting with them, induced participants to like the robots more or less. For example, in Japan, a robot that draws attention to itself may be seen as more individual, more disruptive, and less likeable, whereas in the USA, people may not have as many negative associations with calling attention to one's individuality [30]. Future research could explore this relationship to help determine how to design robots that will be liked depending on preferences for individuality.

\section{Limitations and Future Work}

The Number manipulation in this study included only one or three robots. Under the discontinuity effect [10,31], people respond similarly to three humans as to eight or more humans, suggesting that the responses to groups of robots in this study may generalize to larger groups. However, future research should empirically test if the discontinuity effect applies to robots.

The Behavior manipulation in this study contained only specifically defined "social" and "functional" nonverbal behaviors. Future research should examine other operational definitions of social behavior and other ways for the robots to interact with each other, potentially including verbal behavior. Future research might also examine how believable robots' social behaviors were, given the contexts.

This study was based on responses to only one type of robot, the minimally social STB. Future research should examine if people respond similarly to Number and Behavior of other types of robots (e.g., mechanomorphic, humanoid).
In this study, STBs were placed in two Countries: Japan and the USA. Although the results suggest that people had similar explicit (survey) responses in these countries, further research should examine responses in other countries. The study also occurred in university cafeterias, resulting in many students as participants. Results may differ if younger, older, or differently educated participants were used.

\section{Design Recommendations}

Our results show that, in both Japan and the USA, people tend to interact with robot groups and to use them for their intended purpose (collecting trash) with greater frequency than they do with single robots. However, the number of robots present on its own did not influence people's attitudes toward robots, rather it interacted with robot behavior. For functional robots, group interaction carried more positive consequences in terms of participant attitude and behavior than might have been expected. Applications that use functional robots in groups may not require much concern regarding group effects. For robots displaying social cues, however, more explicit care will be needed to ascertain how to make people more comfortable with robot groups. These could involve the use of prejudice reduction strategies, such as initial positive contact with an individual robot, or less overt socialization of the robots with each other to minimize the impression that they are a cohesive entity. More work is needed to ascertain the best way for robots to engage with groups of people, which our study suggests might have a beneficial effect on HRI. As most robots are evaluated with individuals, and designed to interact with individuals, our study indicates the need to pay more attention to human-robot group interaction in the future.

\section{CONCLUSION}

In this study, Sociable Trash Box (STB) robots were placed in university cafeterias. When the robots were in groups, people interacted with them more than with single robots and reported similar levels of liking. When the robots behaved socially, rather than functionally, participants reported them to be friendlier and more helpful. Japanese participants reported liking the robots more than USA participants. Stronger than any main effect was the interaction across conditions. Regardless of country, participants preferred single social robots and group functional robots to single functional and group social robots. Behaviorally, Japanese participants looked at and interacted more with robots they did not like than with robots they did like, but USA participants did the opposite. This suggests that researchers and designers should be aware of how robot characteristics influence group effects.

\section{ACKNOWLEDGMENTS}

Thank you to Kazuya Sata, Catherine Sembroski, Kartik Adur, and Matthew Francisco for help with the STBs. Thank you to the University staff in Toyohashi and Indiana for allowing our robots to roam the cafeterias. Thanks also to Daryl Hanson for his comments on early versions of this study. 


\section{REFERENCES}

[1] R. A. Brooks, "Flesh and machines: How robots will change us." 2002: Pantheon Books New York.

[2] D. Kuchenbrandt, et al., "When a Robot's Group Membership Matters," International Journal of Social Robotics, 2013. 5(3): 409-417.

[3] M. R. Fraune and S. Šabanović, "Negative Attitudes toward Minimalistic Robots with Intragroup Communication Styles," IEEE ROMAN 2014, the 23rd IEEE International Symposium on Robot and Human Interactive Communication, 2014.

[4] W. L. Chang, et al., "The effect of group size on people's attitudes and cooperative behaviors toward robots in interactive gameplay," in ROMAN, 2012 IEEE. 2012. IEEE.

[5] N. Bee, E. André, and S. Tober, "Breaking the ice in human-agent communication: Eye-gaze based initiation of contact with an embodied conversational agent. in Intelligent Virtual Agents," Springer, 2009.

[6] L. Gong, "How social is social responses to computers? The function of the degree of anthropomorphism in computer representations," Computers in Human Behavior, 2008. 24(4): 1494-1509.

[7] M. R. Fraune, et al., "Rabble of Robots Effects: Number and Type of Robots Modulates Attitudes, Emotions, and Stereotypes," Proceedings of the International Conference on Human Robot Interaction (HRI), 2015. ACM/IEEE.

[8] S. Paepcke, and L. Takayama, "Judging a bot by its cover: an experiment on expectation setting for personal robots," Human-Robot Interaction (HRI), 2010 5th ACM/IEEE International Conference on. 2010. IEEE.

[9] D. Kuchenbrandt, et al., "Minimal group-maximal effect? evaluation and anthropomorphization of the humanoid robot NAO," Social Robotics. 2011, Springer. pp. 104-113.

[10] H. Tajfel, et al., "Social categorization and intergroup behaviour. European journal of social psychology," 1971. 1(2): 149-178.

[11] P. H. Kahn Jr, et al, "Do people hold a humanoid robot morally accountable for the harm it causes?" Proceedings of the seventh annual ACM/IEEE international conference on Human-Robot Interaction. 2012. ACM.

[12] T. Fong, I. Nourbakhsh, and K. Dautenhahn, "A survey of socially interactive robots," Robotics and Autonomous Systems, 2003. 42(3): 143-166.

[13] J. Goetz, S. Kiesler, and A. Powers, "Matching robot appearance and behavior to tasks to improve human-robot cooperation," Robot and Human Interactive Communication, 2003. Proceedings. ROMAN 2003. The 12th IEEE International Workshop on. 2003. IEEE.

[14] M. K. Lee, S. Kiesler, and J. Forlizzi, "Receptionist or information kiosk: how do people talk with a robot?" Proceedings of the 2010 ACM conference on Computer supported cooperative work. 2010. ACM.

[15] F. Kaplan, "Who is afraid of the humanoid? Investigating cultural differences in the acceptance of robots," International Journal of Humanoid Robotics, 2004. 1(03): 465-480.
[16] R. M. Geraci, "Spiritual robots: Religion and our scientific view of the natural world," Theology and Science, 2006. 4(3): 229-246.

[17] D. S. Syrdal, et al., "Examining the frankenstein syndrome," Social Robotics. 2011, Springer. pp. 125-134.

[18] C. Bartneck, et al., "A cross-cultural study on attitudes towards robots," HCI International. 2005.

[19] D. S. Syrdal, T. Nomura, and K. Dautenhahn, "The Frankenstein Syndrome Questionnaire-Results from a Quantitative Cross-Cultural Survey," Social Robotics. 2013, Springer. pp. 270-279.

[20] H. R. Lee, et al., "Cultural design of domestic robots: A study of user expectations in Korea and the United States," RO-MAN, 2012 IEEE. 2012. IEEE.

[21] H. R. Lee and S. Šabanović, "Culturally variable preferences for robot design and use in South Korea, Turkey, and the United States," Proceedings of the $2014 \mathrm{ACM} / \mathrm{IEEE}$ International Conference on Human-Robot Interaction. 2014. ACM.

[22] D. Li, P.P. Rau, and Y. Li, "A cross-cultural study: effect of robot appearance and task." International Journal of Social Robotics, 2010. 2(2): $175-186$.

[23] M. B. Brewer. and Y.-R. Chen, "Where (who) are collectives in collectivism? Toward conceptual clarification of individualism and collectivism," Psychological review, 2007. 114(1): 133.

[24] J. Jetten, et al., "Differentiation between and within groups: The influence of individualist and collectivist group norm.". European Journal of Social Psychology, 2006. 36(6): 825-843.

[25] W. L. Chang, S. Šabanović, and L. Huber, "Use of seal-like robot PARO in sensory group therapy for older adults with dementia," Proceedings of the 8th ACM/IEEE international conference on Human-robot interaction. 2013. IEEE Press.

[26] T. Kanda, et al., "An affective guide robot in a shopping mall." HumanRobot Interaction in Social Robotics, 2012: pp. 52.

[27] P. M. Todd, S.S. Place, and R.I. Bowers, "Simple heuristics for mate choice decisions," Social Judgment and Decision Making, 2012: pp. 193-207.

[28] Yamaji, Y., et al., Stb: Child-dependent sociable trash box. International Journal of Social Robotics, 2011. 3(4): p. 359-370.

[29] Cesario, J. and C.D. Navarrete, "Perceptual Bias in Threat Distance The Critical Roles of In-Group Support and Target Evaluations in Defensive Threat Regulation," Social Psychological and Personality Science, 2014. 5(1): p. 12-17.

[30] Y. Gorodnichenko and G. Roland, "Understanding the individualismcollectivism cleavage and its effects: Lessons from cultural psychology," Institutions and Comparative Economic Development, 2012. 150: pp. 213.

[31] T. Wildschut, et al., "Beyond the group mind: a quantitative review of the interindividual-intergroup discontinuity effect," Psychological Bulletin, 2003. 129(5): p. 698 